\title{
Fuzzy PID Iterative learning control for a class of Nonlinear Systems with Arbitrary Initial Value \\ Xiaohong Hao and Dongjiang Wang
}

School of Computer and communication. Lanzhou University of technology, Lanzhou 730050, China

Keywords: Nonlinear system; Arbitrary initial value; Fuzzy PID Iterative learning control; Norm theory. Operator theory

\begin{abstract}
Aiming at a class of nonlinear systems running repeatedly in the study of the convergence of iterative learning control, a PID fuzzy iterative learning control algorithm has been proposed in the arbitrary initial value using fuzzy PID control algorithm, using traditional experience PID parameters by reference, and experiencing real-time correction of PID parameters, generating higher precision fuzzy PID learning law. The use of operator theory and the theory of convergence norm has been proved by simulation experiment, nonlinear systems under arbitrary initial conditions, the use of fuzzy PID iterative learning control algorithm, the system converges faster, higher tracking accuracy, the output of the error curve faster tends to zero. The results of the demonstrates the effectiveness of the algorithm.
\end{abstract}

\section{Introduction}

Iterative learning control is a branch of intelligent control. It has a good effect on the research of non-linear and strong-coupling control systems with repetitive motion. By constantly adjusting the input of the control system, the tracking performance of tracking system is improved. A complete tracking of the desired output by a controlled object over a finite time interval. PID control, robust control and optimal control cannot achieve high-precision tracking control because of the uncertainties such as initial error and initial state deviation, which affect most system modeling accuracy.

Arimoto [1] and others have studied the convergence of the iterative learning control system to a certain range of guesses, but cannot guarantee that the learning law converges to the true value. Lee [2] and others gave the D-type, PD-type iterative learning control convergence to a certain extent. Chen [3] for a given iterative learning control system for the initial value of the problem, through the system input and initialize the system to achieve the same time iterative tracking. Sun Mingxuan et al. [4] discussed the problem of convergence for a given D-type PD-type iterative control system when the initial value of the system is biased. Ren Xuemei, etc. [5] gives the simultaneous input and initial value of learning, the initial state of the system at the beginning of learning without the request, the results show that the feasibility of this method. Yao Zhongshu et al [5] proposed the iterative learning controller in the frequency domain in the design of the idea, given in any initial iterative learning control algorithm under the sufficient conditions for convergence. Lu et al. [6] studied the iterative learning control of the effect of accelerating the suppression of random initial state errors. Sun et al [7] studied the open-closed-loop PD-type iterative learning control for nonlinear time-delay systems.

In order to improve the control precision of the controlled system and accelerate the convergence speed in the iteration domain, the iterative learning control and the fuzzy control are combined to optimize the parameters of the iterative learning controller in order to achieve better control effect on the nonlinear and uncertain complex control system. Fuzzy control is difficult to establish an accurate mathematical model of the system has strong robustness; many scholars will iterative learning control and fuzzy control combined to achieve a more ideal control effect. Pok [8] proposed a fuzzy iterative learning control algorithm, the algorithm will be filtered after the previous iteration control signal, and with the current error and error accumulation of the derivative as fuzzy iterative learning controller output, simulation experiments verify that the new The algorithm improves the accuracy of system tracking. Yu [9] used iterative learning algorithm to 
modify the fuzzy control rules. Zhang Liping [10] proposed a closed-loop iterative learning controller for nonlinear dynamic model combining fuzzy-fuzzy control and iterative learning control, and improved the fuzzy control Smoothness of the nonlinear mapping, and has good robustness.

The traditional PID iterative learning control controller is not ideal for nonlinear system time-varying systems and time-delay systems. In order to solve this problem, a nonlinear controller is needed. In this paper, a fuzzy controller based on fuzzy-PID control is proposed to solve the above problems. In this paper, a fuzzy controller based on fuzzy-PID is proposed, which is a nonlinear controller for controlling complex systems. Algorithm, using the traditional empirical PID parameters as a reference, and real-time correction of empirical PID parameters to generate a more accurate fuzzy PID learning law. So that the system convergence faster, higher tracking accuracy.

\section{ILC Problem Description}

The dynamic equations of a class of nonlinear time-varying systems are given as follows: $\left\{\begin{array}{l}x(\mathrm{t})=f(x(t), t)+B(t) u(t) \\ y(t)=g_{x}(t, x(t))\end{array}\right.$

among $t \in[0, T], x(t) \in R^{n}$ Is the state quantity, ${ }^{u(t) \in R^{r}}$ For the control volume, ${ }^{y(t) \in R^{m}}$ For the output $f, B, C$ Is the matrix of the corresponding dimension, Let Iterative learning control system initial value of each iteration arbitrary, In time $t \in[0, T]$,Applying PID Type Iterative Learning Law, By adjusting the control input $u_{k}(t)$, So that the output sequence $\left\{y_{k}(t)\right\}$ Consistent convergence in the desired trajectory.

In the times iteration, the system equation is expressed as:

$$
\begin{aligned}
& x_{k}(t)=f(x(t), t)+B(t) u_{k}(t) \\
& y_{k}(t)=g_{x}\left(t, x_{k}(t)\right)
\end{aligned}
$$

Fuzzy PID iterative learning law is as follows:

$$
\begin{aligned}
& u_{k+1}(t)=u_{k}(t)+\left(\Gamma+k_{p}\right) e_{k+1}(t)+\left(\Phi+k_{i}\right) \\
& \int e_{k+1}(t) d(t)+\left(\Psi+k_{d}\right) \frac{d\left(e_{k}(t)\right)}{d(t)}
\end{aligned}
$$

In formula, for the learning gain matrix, the output error is:

$e_{k}(t)=y_{d}(t)-y_{k}(t)$

The system initial state adopts the iterative learning law:

$x_{k+1}(0)=x_{k}(0)+B(0) L(0) e_{k}(0)$

\section{Fuzzy PID Iterative Learning Control}

Fuzzy PID Controller. In order to improve the precision of system control and obtain good control effect for smooth operation, a fuzzy PID iterative learning control algorithm is adopted. The traditional empirical PID parameters are used as reference, and the empirical PID parameters are corrected in real time to generate more accurate Fuzzy logic learning law, so that the system has a good dynamic tracking performance, and improve the iterative convergence rate of the system. PID fuzzy parameter is to find the three parameters of PID fuzzy relationship between E and EC in the running by constantly testing $\mathrm{E}$ and $\mathrm{EC}$, according to the fuzzy principle of the three parameters modified to meet the different $\mathrm{E}$ and $\mathrm{EC}$ when the Control parameters required.

1. Proportional link $k_{P}$ 
The effect of proportional coefficient is to speed up the response speed of the system. The higher the response speed of the system, the faster the response speed of the system, the higher the adjustment accuracy of the system, but easy to produce overshoot, and even lead to system instability. The value is too small, it will reduce the adjustment accuracy, so that the corresponding slow, thereby extending the adjustment time.

\section{Integral link $k_{i}$}

The effect of the integral coefficient is to eliminate the steady-state error of the system. The higher the integral coefficient is, the faster the static error is eliminated, but if it is too large, the integral saturation will occur at the beginning of the response process, which will cause the overshoot of the response process. If too small, will make the system static error is difficult to eliminate, affect the system regulation accuracy.

\section{Differential link $k_{d}$}

The role of differential coefficient is to improve the dynamic performance of the system, its role in the response process to suppress deviation in other directions change, and will reduce the system's interference performance. It can display the variation of the signal deviation, and can introduce a correction amount in the course of the deviation change to control the system action, and prevent the adjustment time increase when the deviation is too large, thus effectively reducing the adjustment time of the system

The Fuzzy Control Rule Set Is Established. The fuzzy control rule table shown in the table is established, and the corresponding fuzzy variable is determined according to the definition in the table. The core of the design of fuzzy control is the technical knowledge and practical experience of the designer, establish the appropriate fuzzy rules table, get the fuzzy rules of three parameters table. Define the fuzzy control input and output fuzzy subset are $\{N B, N M, N S, Z E, P S, P M, P B\}$

(1)The fuzzy rule table of $k_{p}$

Table $2.1 \quad k_{p}$ fuzzy rule table

\begin{tabular}{llllllll}
\hline & $N B$ & $N M$ & $N S$ & $Z O$ & $P S$ & $P M$ & $P B$ \\
\hline NB & PB & PB & PM & PM & PS & ZO & ZO \\
NM & PB & PB & PM & PS & PS & ZO & NS \\
NS & PM & PM & PM & PS & ZO & NS & NS \\
ZO & PM & PM & PS & ZO & NS & NM & NM \\
PS & PS & PS & ZO & NS & NS & NM & NM \\
PM & PS & ZO & NS & NM & NM & NM & NB \\
PB & ZO & ZO & NM & NM & NM & NB & NB \\
\hline
\end{tabular}

(2)The fuzzy rule table of $k_{i}$

Table $2.2 \quad k_{i}$ fuzzy rule table

\begin{tabular}{llllllll}
\hline & $N B$ & $N M$ & $N S$ & $Z O$ & $P S$ & $P M$ & $P B$ \\
\hline NB & NB & NB & NM & NM & NS & ZO & ZO \\
NM & NB & NB & NM & NS & NS & ZO & ZO \\
NS & NM & NM & NS & NS & ZO & PS & PS \\
ZO & NM & NM & NS & ZO & PS & PM & PM \\
PS & NS & NS & ZO & PS & PS & PM & PB \\
PM & ZO & ZO & PS & PS & PM & PB & PB \\
PB & ZO & ZO & PS & PM & PM & PB & PB \\
\hline
\end{tabular}

(3)The fuzzy rule table of $k_{d}$ 
Table $2.3 \quad k_{d}$ fuzzy rule table

\begin{tabular}{llllllll}
\hline & $N B$ & $N M$ & $N S$ & $Z O$ & $P S$ & $P M$ & $P B$ \\
\hline NB & PS & NS & NB & NB & NB & NM & PS \\
NM & PS & NS & NB & NM & NM & NS & ZO \\
NS & ZO & NS & NM & NM & NS & NS & ZO \\
ZO & ZO & NS & NS & NS & NS & NS & ZO \\
PS & ZO & ZO & ZO & ZO & ZO & ZO & ZO \\
PM & PB & NS & PS & PS & PS & PS & PB \\
PB & PB & PM & PM & PM & PS & PS & PB \\
\hline
\end{tabular}

$k_{p}, k_{i}, k_{d}$ The fuzzy rule table is established,According to the following method According to the fuzzy variables, according to the input and output variables of the fuzzy controller, adjust the gain factor continuously. Fuzzy rules to determine, determine the membership function, and then use the center of gravity to its fuzzy solution, the fuzzy PID controller can be output parameters:

$$
k_{p}=\frac{\sum_{q=1}^{n} \mu_{k_{p}}\left(\Delta k_{p}\right) \Delta k_{p}}{\sum_{q=1}^{n} \mu_{k_{p}}\left(\Delta k_{p}\right)} k_{i}=\frac{\sum_{i=1}^{n} \mu_{k_{i}}\left(\Delta k_{i}\right) \Delta k_{i}}{\sum_{i=1}^{n} \mu_{k_{i}}\left(\Delta k_{i}\right)} k_{d}=\frac{\sum_{d=1}^{n} \mu_{k_{d}}\left(\Delta k_{d}\right) k_{d}}{\sum_{d=1}^{n} \mu_{k_{d}}\left(k_{d}\right)}
$$

Is the membership function of the output, $\Delta k_{p}, \Delta k_{i}, \Delta k_{d}$ As the output of the fuzzy variable, ${ }^{k_{p}}, k_{i}, k_{d}$ Is the amount of output after defuzzification. The resulting fuzzy learning law is: $u_{k+1}(t)=u_{k}(t)+\left(\Gamma+k_{p}\right) e_{k+1}(t)+\left(\Phi+k_{i}\right)$

$$
\int e_{k+1}(t) d(t)+\left(\Psi+k_{d}\right) \frac{d\left(e_{k}(t)\right)}{d(t)}
$$

\section{Convergence Analysis}

Before proof of convergence, the following definitions and lemmas are introduced

$$
\begin{aligned}
& \text { definition } 1 \text { Vector function } h:[0, T] \rightarrow R^{n} \text { Is defined as }\|h\|_{\lambda}=\sup \{\exp (-\lambda t) \| h(t)\}, \lambda>0 \text {. } \\
& \|\cdot\|_{\text {is } R^{n}}
\end{aligned}
$$

On a norm.

lemma 1:For vector functions $h:[0, T] \rightarrow R^{n}$,if $\|h(t)\|_{\lambda}=\int_{0}^{\tau} f(\tau) d \tau$, then

$\|h(t)\|_{\lambda} \leq 1-\exp (-\lambda t)\|f(t)\|_{\lambda}$

lemma 2: Set the constant sequence $\left\{b_{k}\right\}_{k \geq 0}\left(b_{k} \geq 0\right)$ Convergence to zero.

operator $k \rightarrow \infty$ Satisfy:

$$
\left\|Q_{k}(u)(t)\right\| \leq M\left(b_{k}+\int_{0}^{t}\|u(s)\| d s\right)
$$

among $M \geq 1$ Is constant, $C_{r}[0, T]$ Function space of continuous function, and the dimension vector takes the maximum norm.

$P(u)(t)=p(t) u(t)$.

then $\lim \left(P+Q_{n}\right)\left(P+Q_{n-1}\right) \cdots\left(P+Q_{0}\right)(u)(0)=0$

theorem 1 For the nonlinear system described by equation (1),

when $t \in[0, T]$ The following conditions are satisfied:

when $k \rightarrow \infty, y_{k}(t)$ Converge uniformly to $y_{d}(t)$ 。

prove: via(1), (3), (5) 
$=x_{k}(0)+B(0) L(0) e_{k}(0)+\int_{0}^{t}\left[f\left(\tau, x_{k+1}(\tau)-f\left(\tau, x_{k}(\tau)\right] d \tau-\quad \int_{0}^{t} \frac{d\left[B(\tau)\left(L(\tau)+k_{d}\right)\right]}{d \tau} e_{k}(\tau) d \tau-\int_{0}^{t} B(\tau)\left(H(\tau)+k_{p}\right) e_{k}(\tau) d \tau\right.\right.$ $+t\left(P(t)+k_{i}\right) \int_{0}^{t} e_{k}(\tau) d \tau$

By the differential mean value theorem available:

$$
\begin{aligned}
& e_{k+1}(t)-e_{k}(t)=\left(y_{d}(t)-y_{k+1}(t)\right)-\left(y_{d}(t)-y_{k}(t)\right) \\
& =g\left(t, x_{k}(t)\right)-g\left(t, x_{k+1}(t)\right) \\
& =g_{x}\left(t, \varepsilon_{k}(t+1)\left(x_{k}(t)-x_{k+1}(t)\right)(8)\right. \\
& \text { 又 } e_{k+1}(t)-e_{k}(t)=-g_{x}\left(t, \varepsilon_{k}(t)\right) B(t)\left(L(t)+k_{d}\right) e_{k}(t)-g_{x}\left(t, \varepsilon_{k}(t)\right) \int_{0}^{t}\left[f \left(\tau, x_{k+1}(\tau)-f\left(\tau, x_{k}(\tau)\right] d \tau\right.\right. \\
& +g_{x}\left(t, \varepsilon_{k}(t)\right)\left\{\int_{0}^{t} \frac{d\left[B(\tau)\left(L(\tau)+k_{d}\right)\right]}{d \tau} e_{k}(\tau) d \tau-\int_{0}^{t} B(\tau)\left(H(\tau)+k_{p}\right) e_{k}(\tau) d \tau\right\} \\
& +g_{x}\left(t, \varepsilon_{k}(t)\right) t\left(P(t)+k_{i}\right) \int_{0}^{t} e_{k}(\tau) d \tau \\
& \text { 则 } e_{k+1}(9)=\left[I-g_{x}\left(t, \varepsilon_{k}(t)\right) B(t)\left(L(t)+k_{d}\right)\right] e_{k}(t) \\
& -g_{x}\left(t, \varepsilon_{k}(t)\right) \int_{0}^{t}\left[f \left(\tau, x_{k+1}(\tau)-f\left(\tau, x_{k}(\tau)\right] d \tau\right.\right. \\
& +g_{x}\left(t, \varepsilon_{k}(t)\right)\left\{\int_{0}^{t} \frac{d\left[B(\tau)\left(L(\tau)+k_{d}\right)\right]}{d \tau} e_{k}(\tau) d \tau\right. \\
& \left.-\int_{0}^{t} B(\tau)\left(H(\tau)+k_{p}\right) e_{k}(\tau) d \tau+t\left(P(t)+k_{i}\right) \int_{0}^{t} \int_{0}^{t} e_{k}(\tau) d \tau\right\}
\end{aligned}
$$

Define operators $P: C_{r}[0, T] \rightarrow C_{r}[0, T]$ is $p\left(e_{k}(t)\right)=\left[I-g_{x}\left(t, \varepsilon_{k}(t)\right) B(t)\left(L(t)+k_{d}\right)\right] e_{k}(t)(11)$

Known by the known conditions, the spectral radius of $\quad p(t)$ is less than 1.

$Q_{k}\left(e_{k}\right)(t)=g_{x}\left(t, \varepsilon_{k}(t)\right)\left\{\int_{0}^{t} \frac{d\left[B(\tau)\left(L(\tau)+k_{d}\right)\right]}{d \tau} e_{k}(\tau) d \tau\right.$

$$
\left.-\int_{0}^{t} B(\tau)\left(H(\tau)+k_{p}\right) e_{k}(\tau) d \tau\right\}-g_{x}\left(t, \varepsilon_{k}(t)\right) \int_{0}^{t}\left[f \left(\tau, x_{k+1}(\tau)-f\left(\tau, x_{k}(\tau)\right] d \tau\right.\right.
$$

$+g_{x}\left(t, \varepsilon_{k}(t)\right) t\left(P(\tau)+k_{i}\right) \int_{0}^{t} e_{k}(\tau) d \tau$

Equation 11 becomes $e_{k+1}(t)=P e_{k}(t)+Q\left(e_{k}\right)(t)=\left(P+Q_{n}\right)\left(P+Q_{n-1}\right) \cdots\left(P+Q_{0}\right) e_{0}(t)(13)$

The first half of the equation (9) takes the norm

$$
\| g_{x}\left(t, \varepsilon_{k}(t)\right)\left\{\int_{0}^{t} \frac{d\left[B(\tau)\left(L(\tau)+k_{d}\right)\right]}{d \tau} e_{k}(\tau) d \tau-\int_{0}^{t} B(\tau)(H(\tau)\right.
$$
$\left.e_{k}(\tau) d \tau\right\}\|\cdot\| g_{x}\left(t, \varepsilon_{k}(t)\right) \| \cdot t \int_{0}^{t}\left(P(\tau)+k_{i}\right) e_{k}(\tau) d \tau+k_{p}$

$\left\|g_{x}\left(t, \varepsilon_{k}(t)\right)\right\| \cdot \int_{0}^{t}\|B(\tau)\| \cdot\left\|\left(H(\tau)+k_{p}\right)\right\| \cdot\left\|e_{k}(\tau)\right\| d \tau$

$\leq(\alpha \beta+\beta b h+\beta t p) \int_{0}^{t}\left\|e_{k}(t)\right\| d \tau$

Among them, $\beta=\sup \left\|g_{x}\left(t, \varepsilon_{k}(t)\right)\right\| ; \quad h=\sup \left\|H(t)+k_{p}\right\| ; \quad p=\sup \|P(t)\|$

From(1):

$$
\begin{aligned}
& x_{k+1}(t)-x_{k}(t)=\int_{0}^{t}\left[f \left(\tau, x_{k+1}(\tau)-f\left(\tau, x_{k}(\tau)\right] d \tau\right.\right. \\
& +B(t) L(t) e_{k}(t)-\int_{0}^{t} \frac{d\left[B(\tau)\left(L(\tau)+k_{d}\right)\right]}{d \tau} d \tau \\
& +\int_{0}^{t} B(\tau) H(\tau) e_{k}(\tau) d \tau-t \int_{0}^{t}\left(P(\tau)+k_{i}\right) e_{k}(\tau) d \tau
\end{aligned}
$$

On both ends of the norm is: $\left\|x_{k+1}(t)-x_{k}(t)\right\| \leq k_{f} \int_{0}^{t}\left\|x_{k+1}(\tau)-x_{k}(\tau)\right\| d \tau$ $+b l\left\|e_{k}(\tau)\right\|+\alpha \int_{0}^{t}\left\|e_{k}(\tau)\right\| d \tau+b h \int_{0}^{t}\left\|e_{k}(\tau)\right\| d \tau$ 
$+p t \int_{0}^{t}\left\|e_{k}(\tau)\right\| d \tau \leq k_{f} \int_{0}^{t}\left\|x_{k+1}(\tau)-x_{k}(\tau)\right\| d \tau$

$+\left\|e_{k}(\tau)\right\|+(\alpha+b h+p t) \int_{0}^{t}\left\|e_{k}(\tau)\right\| d \tau$

Among them, $\quad \alpha_{1}=\alpha+b h+p t ; l=\sup \left\|l(t)+k_{d}\right\| ; h=\sup \left\|H(t)+k_{p}\right\|$

The type can be written as $\left\|x_{k+1}(t)-x_{k}(t)\right\| \leq k_{f} \int_{0}^{t}\left\|x_{k+1}(\tau)-x_{k}(\tau)\right\| d \tau$

$+b l\left\|e_{k}(\tau)\right\|+\alpha_{1} \int_{0}^{t}\left\|e_{k}(\tau)\right\| d \tau$

$+k_{f} \int_{0}^{t}\left[b l\left\|e_{k}(\tau)\right\| d \tau+\alpha_{1} \int_{0}^{t}\left\|e_{k}(v)\right\| e^{k_{f}(t-\tau)} d v\right.$

Among them, $\quad F=\alpha_{1}+K_{f} b l e^{k_{f} T}+T K_{f \alpha_{1}} e^{k_{f} T}$

The latter half of the norm $\| g_{x}\left(t, \varepsilon_{k}(t)\right) \int_{0}^{t}\left[f\left(\tau, x_{k+1}(\tau)-f\left(\tau, x_{k}(\tau)\right] d \tau \|\right.\right.$

$\leq \beta K_{f} b l \int_{0}^{t}\left\|e_{k}(\tau)\right\| d \tau+\beta K_{f} F \int_{0}^{t} \int_{0}^{\tau}\left\|e_{k}(s)\right\| d s d \tau$

$\leq \beta K_{f} \int_{0}^{t}\left\|x_{k+1}(\tau)-x_{k}(\tau)\right\| d \tau$

$\leq N \int_{0}^{t}\left\|e_{k}(\tau)\right\| d \tau(18)$

among $N=\alpha \beta K_{f} b l+\beta K_{f} F T$

From the above equation $\left\|Q_{k} e_{k}(t)\right\| \leq \alpha \beta \int_{0}^{t}\left\|e_{k}(\tau)\right\| d \tau+\beta b h \int_{0}^{t}\left\|e_{k}(\tau)\right\| d \tau+N \int_{0}^{t}\left\|e_{k}(\tau)\right\| d \tau$ $\leq \sigma\left\|Q_{k} e_{k}(t)\right\| \leq \int_{0}^{t}\left\|e_{k}(\tau)\right\| d \tau$

among $\sigma=\max (1, \alpha \beta+b h \beta+N)$

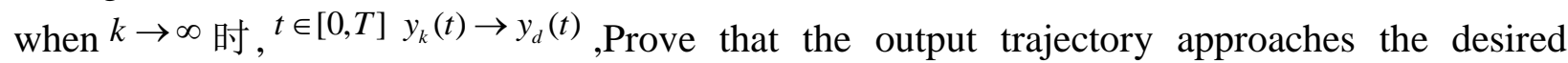
trajectory.

\section{Simulated Analysis}

Consider the following nonlinear system with repetitive motion properties: $\left[\begin{array}{l}x_{1}(t) \\ x_{2}(t)\end{array}\right]=\left[\begin{array}{cc}-2 x_{1}(t)+3 \sin \left(x_{2}(t)\right) & x_{2}(t)+\cos \left(x_{1}(t)\right) \\ 2-3 t & 3-5 t\end{array}\right]$

$\left[\begin{array}{l}x_{1}(t) \\ x_{2}(t)\end{array}\right]+\left[\begin{array}{ll}1 & 0 \\ 5 & 2\end{array}\right]\left[\begin{array}{l}u_{1}(t) \\ u_{2}(t)\end{array}\right]$

$y_{1}(t)=0.5 \cdot x_{1}(t)-x_{2}(t)$

$y_{2}(t)=\cos (\pi t)$

The initial iteration condition is: $\left[x_{1}(0), x_{2}(0)\right]=[1.0,1.0] L(t)=\left[\begin{array}{cc}0.1 & 0 \\ 0 & 0.4\end{array}\right]$

The initial control is $\left[u_{1}(t), u_{2}(t)\right]=[0,1], t \in[0,1]$

Calculated as follows:

$A=I-g_{x}(t, x(t)) B(t) L(t)<1 \quad$ To meet the conditions.

Through matlab simulation available: 


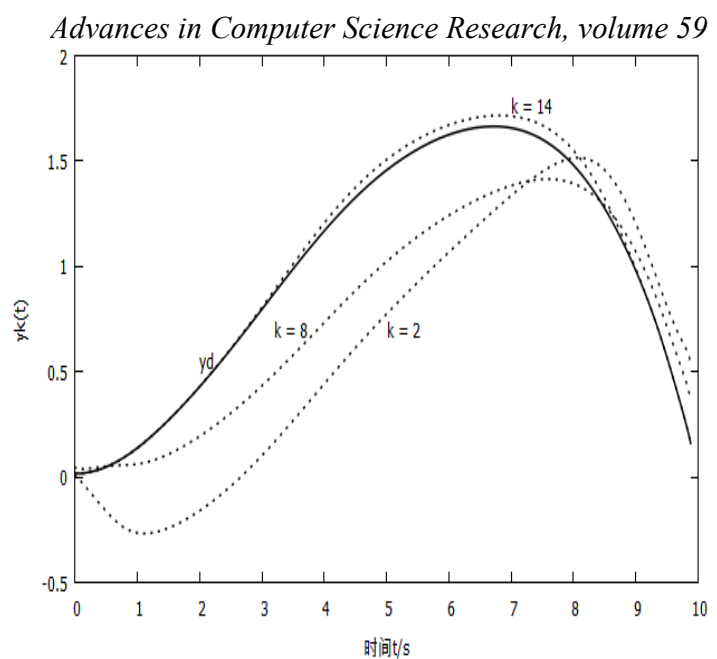

Figure 1. Tracking performance of sine function

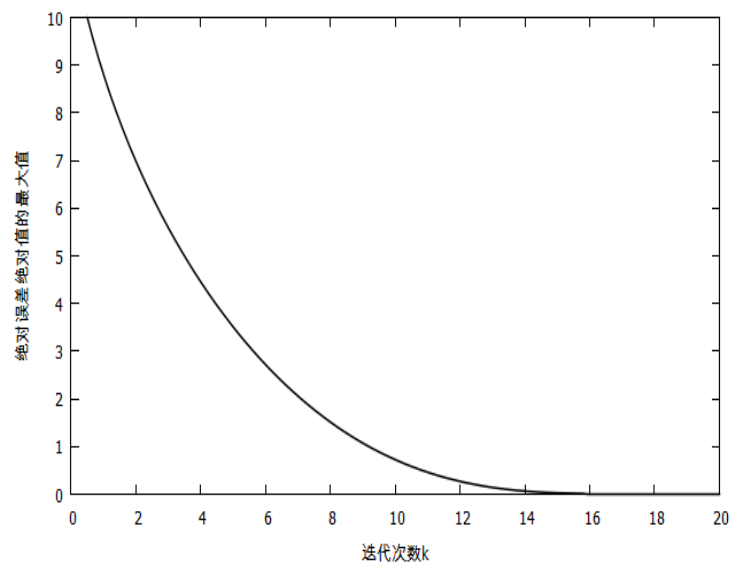

Figure 2. Fuzzy PID type algorithm output error curve

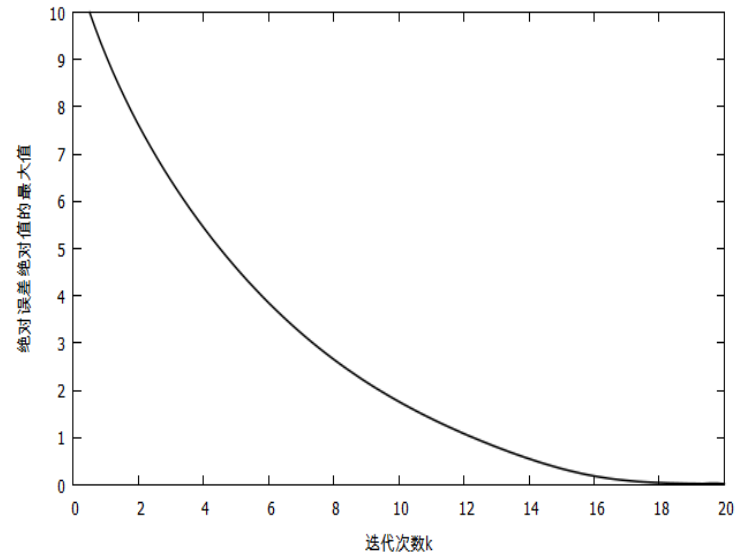

Figure 3. PID type algorithm output error curve

It can be seen from the comparison of Fig. 2 and Fig. 3 that the output error of the system can be approximated to zero by 14 iterations of the fuzzy PID iterative learning control algorithm, while the PD algorithm needs 18 times to reach the same effect.

\section{Concluding Remarks}

In this paper, the fuzzy PID iterative learning control problem for a class of nonlinear systems under arbitrary initial conditions is discussed. It is shown that the convergence condition can be relaxed under any initial learning. Fuzzy PID iterative learning control algorithm is used. Using the traditional empirical PID parameters As a reference, and real-time correction of the empirical PID parameters to generate a more accurate fuzzy learning law of the fuzzy law, without the need for rigorous initial positioning operation can iterate the system to solve the iterative learning control initial value problem, the algorithm Which can track the desired trajectory more quickly and reduce 
PRESS number of iterations to make the systemp have good dynamic tracking performance and improve the convergence speed of the system effectively.

\section{References}

[1] ArimotoS. Robustness of learning control for robot manipulations [J]. Proceedings of IEEE International Conference on Robotics and Automation, Cincinnati, Ohio, USA 1990, 5:1528 1533.

[2] Lee HS,Bien Z. Study on robustness of iterative learning control with non-zero initial error[J]. International Journal ofContro1,1996,64(3):345 359

[3] Sun Mingxuan, Huang Baojian, [3]. PD type iterative learning control for nonlinear systems [J]. Journal of automation, 1998,24 (5): $711 \sim 714$

[4] Chen Y, Wen C, Gong Z and Sun M. An iterative learning controller with initial state learning [J]. IEEE Transactions on Automatic Contro1, 1999, 44(2):371 376.

[5] Ren Xue-mei, Huang Bao-jian,Zhang Xue-zhi,PD-Type Iterative Learning Control Fora Class of Nonlinear System[J]. Acta Automatic Sinica .1998.20(1):74-79(in Chinese)

[6] Owens D H,Hatonen J. Iterative Learning Control-Ari Optimization Paradigm[J]. Annual Reviews in Control. 2005, 29(1): 57-70

[7] Lü Q,Fang Y C, Ren X. Acceleration suppression of random errorson the initial state of iterative learning control, Acta Automatica Sinica,2014; 40( 7) : 1295-1302

[8] Yang X,Sun Y.Closed loop PD-type iterative learning

[9] control of nonlinear delay systems open. Science Technology and Engineering,2011; 11(27):6648-6650

[10]Yu S J,DuanL,Wu J H. Study of fuzzy learning control for electry-hydraulic servo control[C], In: International Conference on Machine Learning and Cybernetics. Xi'an, China: Institute of Electrical and Electronics Engineers Inc,2003, 591-595

[11]Zhang L P, Yang F W. A fuzzy iterative learning control design for point-to-point control of nonlinear system[C]. In: Proceedings of the World Congress on Intelligent Control and Automation. Hangzhou, China: Institute of Electrical and Electronics Engineers Inc, 2004, 246-1249 